\title{
Uncertainties and robustness of the ignition process in type la supernovae
}

\author{
L. Iapichino ${ }^{1}$ and P. Lesaffre ${ }^{2,3}$ \\ 1 Zentrum für Astronomie der Universität Heidelberg, Institut für Theoretische Astrophysik, Albert-Ueberle-Strasse 2, \\ 69120 Heidelberg, Germany \\ e-mail: luigi@ita.uni-heidelberg.de \\ ${ }^{2}$ Laboratoire de Radioastronomie, École Normale Supérieure, 24 rue Lhomond, 75231 Paris Cedex 05, France \\ e-mail: pierre.lesaffre@lra.ens.fr \\ 3 Institute of Astronomy, Madingley Road, Cambridge CB30HA, UK
}

Received 16 September 2009 / Accepted 15 December 2009

\begin{abstract}
Context. It is widely accepted that the onset of the explosive carbon burning in the core of a carbon-oxygen white dwarf (CO WD) triggers the ignition of a type Ia supernova (SN Ia). The features of the ignition are among the few free parameters of the SN Ia explosion theory.

Aims. We explore the role for the ignition process of two different issues: firstly, the ignition is studied in WD models coming from different accretion histories. Secondly, we estimate how a different reaction rate for C-burning can affect the ignition.

Methods. Two-dimensional hydrodynamical simulations of temperature perturbations in the WD core ("bubbles") are performed with the FLASH code. In order to evaluate the impact of the C-burning reaction rate on the WD model, the evolution code FLASH_THE_TORTOISE from Lesaffre et al. (2006, MNRAS, 368, 187) is used.

Results. In different WD models a key role is played by the different gravitational acceleration in the progenitor's core. As a consequence, the ignition is disfavored at a large distance from the WD center in models with a larger central density, resulting from the evolution of initially more massive progenitors. Changes in the $\mathrm{C}$ reaction rate at $T \lesssim 5 \times 10^{8} \mathrm{~K}$ slightly influence the ignition density in the WD core, while the ignition temperature is almost unaffected. Recent measurements of new resonances in the C-burning reaction rate (Spillane et al. 2007, Phys. Rev. Lett., 98, 122501) do not affect the core conditions of the WD significantly.

Conclusions. This simple analysis, performed on the features of the temperature perturbations in the WD core, should be extended in the framework of the state-of-the-art numerical tools for studying the turbulent convection and ignition in the WD core. Future measurements of the C-burning reactions cross section at low energy, though certainly useful, are not expected to affect our current understanding of the ignition process dramatically.
\end{abstract}

Key words. supernovae: general - hydrodynamics - methods: numerical - nuclear reactions, nucleosynthesis, abundances white dwarfs

\section{Introduction}

The sequence of events that leads to the ignition of thermonuclear flames in the core of mass-accreting carbon-oxygen white dwarfs (CO WD) marks the start of the explosion of a type Ia supernova (SN Ia). It is known from theory (Hillebrandt \& Niemeyer 2000) that the ignition process spans over a broad range of typical length and time scales, corresponding to the transition from hydrostatic to explosive C-burning. As a consequence, a proper modeling of this evolutionary phase is very challenging both from the theoretical and computational point of view. In the framework of the single degenerate explosion scenario, a detailed knowledge of the features of the WD at ignition is crucial to determine the initial conditions of the explosion models, i.e. to shape the initial flame morphology in the numerical simulations.

The dependence of the explosion outcome and features on the initial spatial distribution of the flame kernels has been often debated, given the underlying relative freedom in constraining the unknowns of the multi-spot ignition scenario (GarcíaSenz \& Bravo 2005; Röpke et al. 2006b, 2007b). In general it turns out that a larger number of ignition points results in a more vigorous deflagration phase. Interestingly, in recent models with deflagration to detonation transition (DDT), ignition closer to the center leads to a weaker detonation phase and a smaller production of ${ }^{56} \mathrm{Ni}$, because of the stronger WD pre-expansion in the deflagration stage (Woosley et al. 2008; Kasen et al. 2009).

Several different approaches have been used to address the problem of SN Ia ignition. Most of the work on this topic has been devoted to full-star simulations of the CO WD, both using analytical models (Woosley et al. 2004; Wunsch \& Woosley 2004) and numerical simulations (Höflich \& Stein 2002; Kuhlen et al. 2006; Piro \& Bildsten 2008; Piro \& Chang 2008; Zingale et al. 2009). A parallel branch of studies focuses on the role of the convective Urca process for the ignition (Lesaffre et al. 2005a,b, and references therein).

Recently Lesaffre et al. (2006) studied the link between the evolution of the progenitor in its binary system and the ignition conditions of SNe Ia. They found a broad range of densities and temperatures for WD models at ignition, linked to the initial WD mass and the binary accretion history. The effect of the progenitor diversity on the explosion phase still needs to be explored. In particular, a fundamental issue has to be fully addressed, namely the relation between the properties of the 
ignition process and the homogeneity of SNe Ia as a class of objects. Does ignition contribute to the homogeneity of SNe Ia or, on the contrary (cf. Röpke et al. 2006a), is it more intrinsically linked to the residual diversity of Branch-normal SNe Ia?

A complementary contribution to the study of this problem is provided by the small-scale ( $1 \mathrm{~km}$ or less) modeling approach. These models are motivated by analytical calculations (García-Senz \& Woosley 1995; Wunsch \& Woosley 2004) which predict that temperature perturbations ("bubbles"), produced in the WD's core by the turbulent convective flow finally trigger the thermonuclear runaway. The physics of the thermal fluctuation has been addressed by García-Senz \& Bravo (2005) and Zingale \& Dursi (2007), who focus on the transition from hydrostatic to explosive burning and the propagation of the first thermonuclear flames. Iapichino et al. (2006) studied the bubble physics with a series of small-scale 2D simulations. According to their results, the main feature of the bubble evolution is the fragmentation caused by its own buoyant rise motion, which can be considered in a sense as a cooling effect, counteracting the heating from the hydrostatic nuclear burning. Among nuclear burning and hydrodynamical dispersion, the process with the smaller timescale determines the outcome of the bubble, namely if it will trigger a thermonuclear runaway or will be dispersed and cooled down by thermal conduction.

The goal of the small-scale studies is the description of the $\mathrm{SNe}$ Ia ignition scenario in terms of number of flame kernels, size of the ignition region in the WD core, (a)symmetry of the ignition and stochastic behaviors of the process. There is general agreement that the WD convective flow prior to the thermonuclear runaway plays a crucial role on the ignition. It has also been proposed that the flame kernels could tend to cluster spatially, thus evolving in a sort of ignition plume rather than separate flame seeds (e.g. Livne et al. 2005). This idea is closely linked to the flow in the WD convective core, which is likely to develop a dipole feature (Woosley et al. 2004; Wunsch \& Woosley 2004; Kuhlen et al. 2006; Zingale et al. 2009).

The study of Iapichino et al. (2006) explored only the ignition parameters that are inherent to the bubble physics: the initial bubble diameter, the initial bubble temperature, the initial distance of the bubble from the WD center. Though instructive for understanding the small-scale physics of the temperature perturbations, that work could only very generically constrain the large-scale ignition features through a number of additional theoretical assumptions. In the present work, we widen the scope to additional ignition parameters, which can be related to the ignition condition in a more straightforward way. In particular, the bubble simulations developed in Iapichino et al. (2006) will be used to probe the ignition conditions in the WD models presented in Lesaffre et al. (2006). We will show how the large range of ignition densities in the aforementioned WD models can be linked to a significant diversity for the size of the ignition region.

In the second part of the present work, these results will be applied to a largely unaccounted for issue of the ignition process, namely the assessment of the role of experimental uncertainties in the C-burning reaction rates. In a recent study, Spillane et al. (2007) present new reaction rates for the reactions ${ }^{12} \mathrm{C}\left({ }^{12} \mathrm{C}\right.$, $\alpha)^{20} \mathrm{Ne}$ and ${ }^{12} \mathrm{C}\left({ }^{12} \mathrm{C}, \mathrm{p}\right){ }^{23} \mathrm{Na}$, which are higher than previously assumed (Caughlan \& Fowler 1988). Those authors suggest that the new measurements could affect the ignition and even the explosion theory of SNe Ia. We show in this work that the impact of these measurements on the WD evolution to ignition is actually marginal, and by using the WD evolution code by Lesaffre et al. (2006) we make estimates on the role of a hypothetical higher (or lower) rate.
Table 1. Main features of the four WD models used in the simulations.

\begin{tabular}{ccccc}
\hline \hline Model & $T_{\mathrm{c}}\left[10^{8} \mathrm{~K}\right]$ & $\rho_{\mathrm{c}}\left[10^{9} \mathrm{~g} \mathrm{~cm}^{-3}\right]$ & $g\left[10^{9} \mathrm{~cm} \mathrm{~s}^{-2}\right]$ & $r_{\mathrm{b}}[\mathrm{km}]$ \\
\hline 1 & 7.84 & 1.82 & 5.18 & 163 \\
3 & 7.63 & 2.08 & 5.90 & 154 \\
4 & 7.15 & 3.12 & 8.78 & 135 \\
6 & 6.94 & 4.17 & 11.7 & 123 \\
\hline
\end{tabular}

The structure of the paper is the following: in Sect. 2 the simulations will be introduced, with special emphasis on the details related to the WD background models (Sect. 2.2) and the C-burning reaction network (Sect. 2.3). The results are presented in Sect. 3, and the conclusions are drawn in Sect. 4.

\section{Numerical tools}

\subsection{Bubble setup}

The 2D numerical simulations presented in this work have been performed with the FLASH code (v. 2.3; Fryxell et al. 2000), a hydrodynamical, adaptive mesh refinement (AMR) code especially designed for the study of thermonuclear flashes. The simulation setup is very similar to the one described in Iapichino et al. (2006), and we refer the reader to that work for a more detailed account. The most relevant differences with respect to it are presented in the next sections. A 2D bubble is initialized as a temperature perturbation in a pressure equilibrium with a background medium in a computational domain with a size of $5 \times 20 \mathrm{~km}$. The simulations have a Cartesian geometry and were set with a root grid of $[16 \times 64]$ grid cells and four additional AMR levels, leading to an effective grid size of [ $256 \times 1024]$ and an effective spatial resolution of $2 \times 10^{3} \mathrm{~cm}$. The initial bubble diameter is $D=1 \mathrm{~km}$, considered to be a good order-of-magnitude estimate of the size of the temperature perturbations in the turbulent WD convective flow (Woosley et al. 2004). The initial distance of the bubble from the WD center is set to $R=100 \mathrm{~km}$, and the initial temperature contrast $\Delta T / T$ is set to 0.07 at the bubble location. Both values are in the range predicted by Wunsch \& Woosley (2004) for the generation of temperature fluctuations in the WD core, though these authors refer to the contrast to the core temperature rather than to the local temperature. Note that in the framework of our simplified model $R$ and $\Delta T / T$ are not completely independent parameters: taking a slightly bigger contrast at a given $R$ is like considering a bubble that has already travelled and grown from a slightly smaller distance to the center. Therefore, as long as $\Delta T / T$ is relatively small, its value is not a critical issue in the model.

\subsection{WD models}

Unlike Iapichino et al. (2006), we adopt here the WD models from Lesaffre et al. (2006) as background for the simulations. The models are labelled in Table 1 with the number $m$ in the first column, where $m=1,3,4,6$ is related to the initial mass of the WD: $M_{\text {init }}=(0.6+0.1 \times m) M_{\odot}$. All models are for a cooling age of $t_{a}=0.4 \mathrm{Gyr}$ (see Lesaffre et al. 2006). In Table 1 the quantity $T_{\mathrm{c}}$ is the central temperature of the model, $\rho_{\mathrm{c}}$ is the central density and $g$ is the gravitational acceleration at a distance of $103 \mathrm{~km}$ from the WD center. The burning zone radius of the model $r_{\mathrm{b}}$ is defined as the radius within which one-half of the luminosity is generated.

The details of the modeling of the accretion phase are reported in Lesaffre et al. (2006). The models are taken at the time 


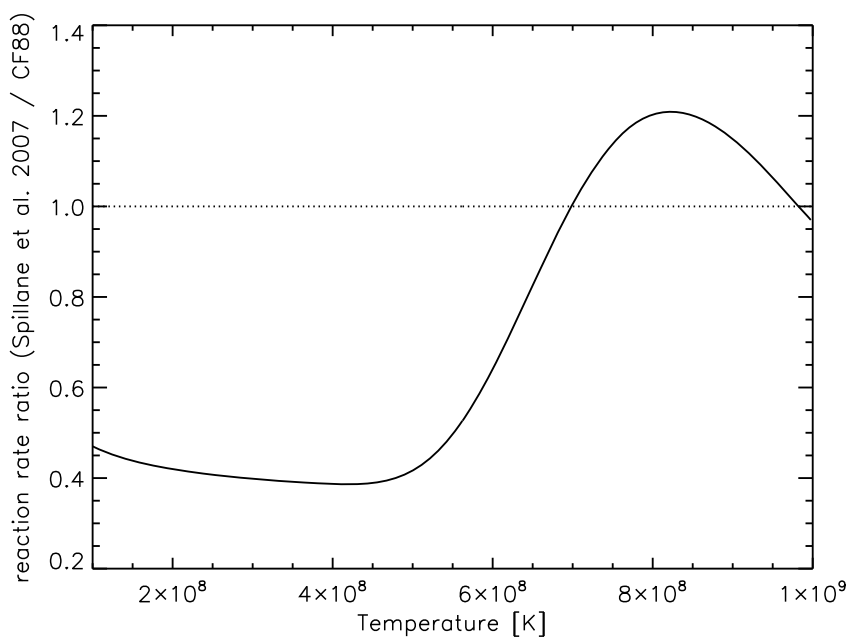

Fig. 1. Ratio (solid line) between the new reaction rate of Spillane et al. (2007) for C-burning and the Caughlan \& Fowler (1988) rate in a temperature range relevant for the ignition of $\mathrm{SNe}$ Ia. The nonresonant behavior at low temperature has been extrapolated from the analytic fit of Spillane et al. (2007), which refer to a higher energy range.

in the accretion history when the burning time scale is equal to the convective turnover time scale (this corresponds to the ignition criterion $\alpha=1$ of Lesaffre et al. 2006, Sect. 2.5). The chemical composition of the WD is not set as in Iapichino et al. (2006) $\left(\mathrm{X}\left({ }^{12} \mathrm{C}\right)=\mathrm{X}\left({ }^{16} \mathrm{O}\right)=0.5\right)$, but is mapped from the WD model. We pick the models from Lesaffre et al. (2006) which use values from Umeda et al. (1999) as initial conditions before the accretion stage. The $\mathrm{C} / \mathrm{O}$ ratio in the $\mathrm{WD}$ core at ignition ends up between 0.74 and 0.46 , depending on the model.

The gravitational acceleration $g(r)$ is derived from the WD mass profile as

$g(r)=\frac{G M(r)}{r^{2}}$,

where $G$ is the gravitational constant and $M(r)$ the mass contained in the spherical shell with the radius $r$. The acceleration is applied to the computational domain as a force pointing downwards along the $y$-axis, constant in space and time.

\subsection{Nuclear burning and carbon reaction rate}

In the initial WD models the abundances of four chemical species are mapped, i.e. ${ }^{12} \mathrm{C},{ }^{16} \mathrm{O},{ }^{22} \mathrm{Ne}$ and ${ }^{24} \mathrm{Mg}$. As in Iapichino et al. (2006), the hydrostatic nuclear burning is followed with the reaction network iso7 (Timmes et al. 2000), which evolves seven $\alpha$-isotopes. As ${ }^{22} \mathrm{Ne}$ is not available in the reaction network, this isotope is substituted by ${ }^{20} \mathrm{Ne}$, as suggested and verified by Dursi \& Timmes (2006). We refer to Timmes et al. (2000) for the nuclear rates in the reaction network with the notable exception of the C-burning, which in some calculations is modified according to the following prescriptions.

Spillane et al. (2007) performed a study of the carbon burning reactions ${ }^{12} \mathrm{C}\left({ }^{12} \mathrm{C}, \alpha\right){ }^{20} \mathrm{Ne}$ and ${ }^{12} \mathrm{C}\left({ }^{12} \mathrm{C}, \mathrm{p}\right){ }^{23} \mathrm{Na}$ using $\gamma$-ray spectroscopy with a $\mathrm{C}$ target with ultra-low hydrogen contamination. The explored energy range spanned from 2.10 to $4.75 \mathrm{MeV}$. The most relevant result is the detection of a new resonance at the very low end of the energy range $\left(E_{R}=2.14 \mathrm{MeV}\right)$.

The effect of this new measurement for the C-burning reaction rate (the sum of the contributions of the $\alpha$ and proton channels) as a function of the temperature is shown in Fig. 1. With respect to the reference value of Caughlan \& Fowler (1988), the reaction rate is increased up to $20 \%$ in the temperature range between 7.0 and $9.8 \times 10^{8} \mathrm{~K}$. For comparison, the Gamow energy for the C-burning reactions is $1.5 \pm 0.3 \mathrm{MeV}$ at $T=5 \times 10^{8} \mathrm{~K}$, and $2.1 \pm 0.4 \mathrm{MeV}$ at $T=8 \times 10^{8} \mathrm{~K}$. The new resonance therefore affects the reaction rate mostly in the late hydrostatic burning stage, just prior to ignition. The data do not provide information about the burning rate at lower temperatures, relevant for the "smouldering phase" of the burning in massive CO WDs $\left(5 \times 10^{7} \mathrm{~K} \lesssim T \lesssim 7 \times 10^{8} \mathrm{~K}\right.$; cf. Nomoto 1982). An extrapolation at these temperatures rests upon the new evaluation of the non-resonant contribution to the reaction rate from Spillane et al. (2007), plus general considerations on the unknown resonance structure below $2.0 \mathrm{MeV}$. Assuming that the observed resonance pattern of the reactions continues in a similar way at lower energies, Spillane et al. (2007) state that their rates at lower temperature represent the nonresonant, lower limits to the true values at $E \simeq 1.5 \mathrm{MeV}$.

On the other hand, it is quite clear that the new rates are not relevant for the explosive nucleosynthesis of SNe Ia, which occurs in a different burning regime (nuclear statistical equilibrium) and at $T>10^{9} \mathrm{~K}$, where the corresponding experimental uncertainties on the astrophysical factor are much better constrained.

The relevance of the new rate in the range $7 \times 10^{8} \mathrm{~K}<T<$ $9.8 \times 10^{8} \mathrm{~K}$ therefore motivates the investigation of its effect on the ignition process in $\mathrm{SNe}$ Ia, which typically occurs at these temperatures.

Due to the restrictions of the $\alpha$-network, the C-burning in iso7 is implemented in a way that the reaction rate takes into account both the $\alpha$ and the proton channel, but the only reaction products are ${ }^{20} \mathrm{Ne}$ and $\alpha$. Since the $\alpha$ channel is dominant in the considered temperature range, this approximation is sensible.

It is worth mentioning that besides the above cited measurements several works in the last decade have been devoted to the theoretical prediction of the C-burning reaction rate. Some of them (Cussons et al. 2002; Itoh et al. 2003) state that resonant screening effects could have a dramatic effect (either suppression or enhancement, respectively, for the two cited papers) on the reaction rate at low energy. Jiang et al. (2007) and Gasques et al. (2007) predict that the fusion cross section has a low-energy hindrance in a range of temperatures and densities which include the conditions in the WD core. Gasques et al. (2007) further compute the SN Ia ignition curve in this case, finding that with the reduced burning rate the ignition criteria will be fulfilled at a larger temperature. All these theoretical expectations will not be addressed explicitly in the present work.

\section{Results}

\subsection{Ignition in different WD models}

Four bubble simulations have been performed, one for each WD background model listed in Table 1 . In these runs, the reaction rate is set as in Caughlan \& Fowler (1988). Figure 2 shows the time evolution of the maximum bubble temperature in the four simulations. The bubble calculations with models 1 and 3 go to thermonuclear runaway, whereas in the cases 4 and 6 the bubble is fragmented by the hydrodynamical instabilities and its own rising motion before reaching the runaway. The typical evolutionary timescales are about $25 \%$ longer than the ones reported in Iapichino et al. (2006), because of the lower ${ }^{12} \mathrm{C}$ abundance in the present work. 


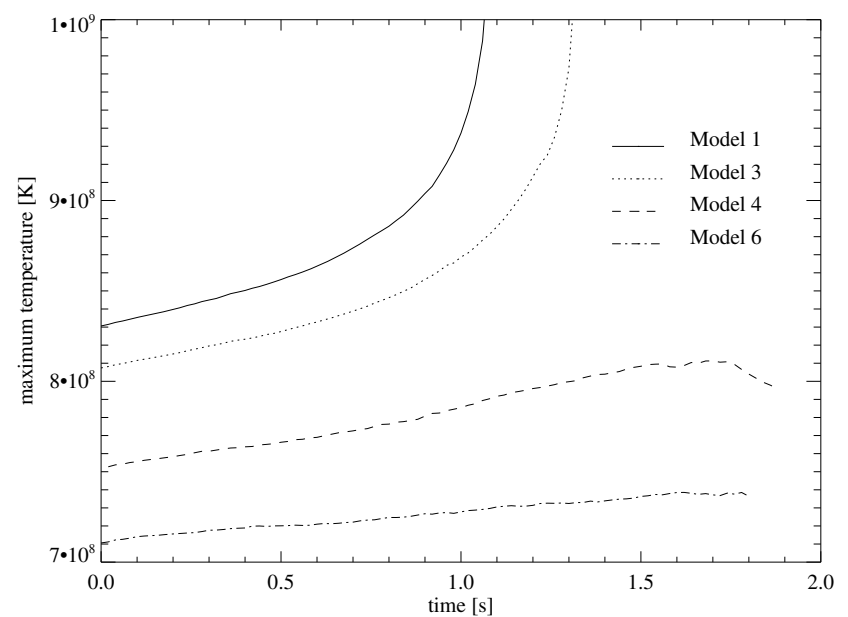

Fig. 2. Maximum bubble temperature as a function of time in four bubble simulations with different WD models reported in the legend. The initial bubble temperature is indicated at $t=0 \mathrm{~s}$, the other bubble parameters are set as described in Sect. 2.1.

Two main points explain the differences in the bubble simulations shown above. First, the initial bubble temperature determines the nuclear timescale. A colder temperature perturbation has a longer ignition timescale $\tau_{\mathrm{n}}$, according to

$\tau_{\mathrm{n}} \propto T^{-22} \rho^{-4}$,

(Woosley et al. 2004; Iapichino et al. 2006) and thus it is dispersed more efficiently by its own buoyant rise.

Secondly, WD models with a larger initial mass are colder and denser in the center and have a larger gravitational acceleration at the initial bubble location (cf. Table 1). This leads to a higher buoyant velocity $v_{\mathrm{b}}$ and thus to smaller dispersion timescales $\tau_{\text {disp }}$ in models 4 and 6 , which can be estimated as

$\tau_{\text {disp }}=\frac{D}{v_{\mathrm{b}}}$,

where $D$ is the bubble diameter.

The ignition condition for a temperature perturbation is met when its nuclear timescale is smaller than the dispersion timescale. In the case of models 4 and 6 , the bubble does not go to thermonuclear runaway: for this set of bubble parameters, the dispersion is faster than the nuclear burning. Suppose that we reduce the bubble distance from the WD center. At the same time the ignition timescale decreases (because the temperature increases) and the dispersion timescale increases (because $g$ decreases towards the center). Both effects make the ignition more likely.

The main result for this part of our study is therefore a dependence of the ignition features on the accreting history of the WD. WDs with a larger initial mass develop a core which is denser and cooler, according to Lesaffre et al. (2006). In these models we find that for a given bubble size the ignition is favored at a smaller distance from the WD center than in models with a smaller initial mass. This interpretation is further strengthened by the computation of the burning zone radius $r_{\mathrm{b}}$ of the WD models (Table 1, fifth column), defined as the radius within which one-half of the luminosity is generated (Woosley et al. 2004). This radius can be considered a good approximation for the size of the region where the temperature perturbations arise. In denser models, $r_{\mathrm{b}}$ is smaller, with a maximum difference between models 1 and 6 of $25 \%$. Temperature perturbations are thus likely to be generated closer to the WD center.

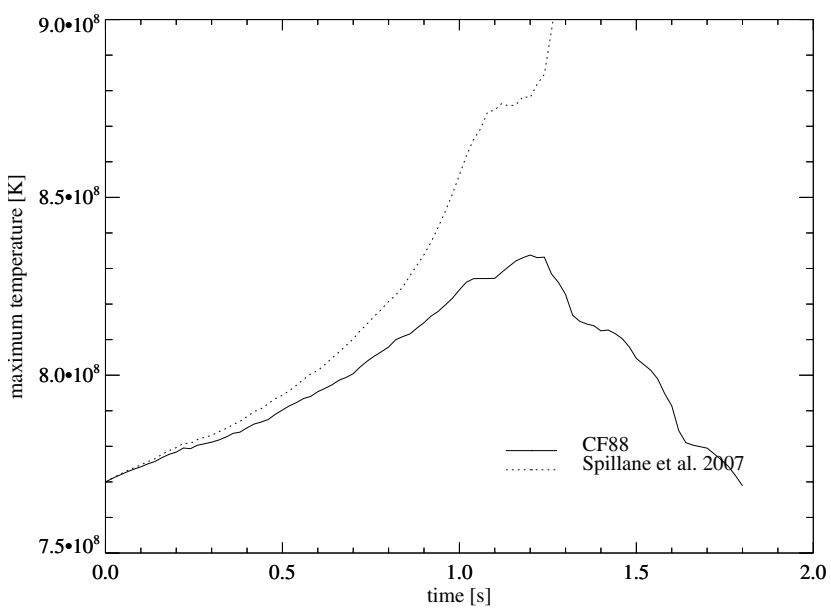

Fig. 3. Maximum bubble temperature as a function of time in two bubble simulations with the same bubble parameters (described in the text) but a different prescription for the reaction rate of C-burning, as indicated in the legend.

\subsection{The role of carbon burning}

The increase of the rate of C-burning in Spillane et al. (2007), though not dramatic, has a relevant impact on the evolution of temperature perturbations due to the high power dependence on $T$ in Eq. (2). This is shown in Fig. 3, which refers to two simulations with the same bubble parameters but different references for the C-burning rate, namely Caughlan \& Fowler (1988) and Spillane et al. (2007). The WD background model is the same as in Iapichino et al. (2006), and the bubble parameters are $T=7.7 \times 10^{8} \mathrm{~K}, D=1 \mathrm{~km}$ and $R=100 \mathrm{~km}$. Not unexpectedly, the bubble simulation with the new reaction rate reaches the thermonuclear runaway, the other does not. From an analytical viewpoint, this is well described by the equation linking the reaction rate $\dot{S}$ and $\tau_{\mathrm{n}}$ (Woosley et al. 2004)

$\tau_{\mathrm{n}}=\left(\frac{1}{\dot{S}} \frac{\mathrm{d} \dot{S}}{\mathrm{~d} t}\right)^{-1} \approx\left(\frac{1}{\dot{S}} \frac{\partial \dot{S}}{\partial T} \frac{\partial T}{\partial t}\right)^{-1}=\frac{C_{\mathrm{p}} T}{23 \dot{S}}$,

where $C_{\mathrm{p}}$ is the specific heat at constant pressure. At $T=$ $8 \times 10^{8} \mathrm{~K}, \dot{S}$ is 1.2 times larger, thus $\tau_{\mathrm{n} \text {,new }} \approx 0.8 \tau_{\mathrm{n}, \mathrm{old}}$, and a smaller nuclear timescale favors the bubble ignition before its fragmentation.

This is not a surprising result and has a limited impact on our knowledge of the ignition process. Another interesting question pertains to the effect of the new rates not only on a temperature perturbation, but on the overall WD structure before ignition. In other words, can a higher rate of C-burning modify the WD models before ignition (Lesaffre et al. 2006) and consequently the features of this process?

In order to investigate this point, we ran two additional WD models with the code used in Lesaffre et al. (2006) (FLASH_THE_TORTOISE ${ }^{1}$ ) under the same conditions as in Model 3, but we varied the adopted rate of C-burning (Table 2). Model 3.1 was run with the rate of Spillane et al. (2007) instead of the Caughlan \& Fowler (1988) rate used for Model 3. The extrapolation of the rate at low temperature is about half the Caughlan \& Fowler (1988) rate in the low temperature range between $10^{8} \mathrm{~K}$ and $5 \times 10^{8} \mathrm{~K}$ (see Fig. 1). As seen in Fig. 4, the central part of the star spends more time at low temperature in that case: as a result the ignition curve is shifted to higher

${ }^{1}$ Code web page: http://astro.ens.fr/?lesaffre. 
Table 2. Main features of the two additional WD models computed to investigate the effects of changing the burning rate (quantities are same as in Table 1).

\begin{tabular}{ccccc}
\hline \hline Model & $T_{\mathrm{c}}\left[10^{8} \mathrm{~K}\right]$ & $\rho_{\mathrm{c}}\left[10^{9} \mathrm{~g} \mathrm{~cm}^{-3}\right]$ & $g\left[10^{9} \mathrm{~cm} \mathrm{~s}^{-2}\right]$ & $r_{\mathrm{b}}[\mathrm{km}]$ \\
\hline 3.1 & 7.68 & 2.20 & 6.23 & 150 \\
$3^{a}$ & 7.63 & 2.08 & 5.90 & 154 \\
3.2 & 7.59 & 2.00 & 5.20 & 164 \\
\hline
\end{tabular}

Notes. ${ }^{(a)}$ As for model 3, the stellar evolution code stalls shortly before the actual criterion for ignition is met, hence these values result of an extrapolation from about $5 \%$ lower temperature models. However, we do not expect the extrapolation errors to change much either the absolute or the relative values of the numbers presented in this table.

densities and the star ignites at a slightly higher density (about $6 \%$ higher, see Table 2). The Spillane et al. (2007) extrapolated burning rate is a lower estimate at low temperature, hence we can take the ignition density value of model 3.1 as an upper estimate. The gravitational acceleration follows the same trend as the central density. On the contrary, the ignition temperature is almost unchanged. This reflects the fact that the reaction rate is an extremely steep function of the temperature. The burning zone radius is almost unchanged as well.

Model 3.2 was run with twice the Caughlan \& Fowler (1988) rate to check how considerable changes in the rate, caused by possible discoveries of new low-lying resonances, can affect the ignition conditions (for example, the Spillane et al. 2007 rate is, at most, only $20 \%$ higher than the Caughlan \& Fowler 1988 rate). Since the rate of burning is now everywhere higher than the Caughlan \& Fowler (1988) rate, the ignition curve on Fig. 4 is shifted to the left hand side and the star ignites at lower density (by about 4\%, as seen on Table 2). As in model 3.1, the ignition temperature is almost unchanged.

To conclude this brief investigation, the main effect of the rate is rather surprising at a low temperature, where it controls the density at which the growth of the convective core starts, and hence determines the future ignition density. The ignition temperature is almost unaffected. The results on ignition temperatures and burning radii qualitatively agree with some calculations done with the heuristic convection model from Wunsch \& Woosley (2004) and the reaction rates described above for an isotropic flow and neglecting nonlinear effects on the burning.

\subsection{Numerical tests on the bubble simulations}

The interpretation of the bubble simulations presented in this work cannot be separated from an evaluation of the numerical issues of the setup. As already discussed in Iapichino et al. (2006), the main features of the bubble physics are the fragmentation due to the buoyant motion, and (if the bubble does not reach the thermonuclear runaway) the subsequent dispersion by heat diffusion. The latter process is expected on length scales $(10-100 \mathrm{~cm})$ which are one to two orders of magnitude smaller than the effective spatial resolution of the performed simulations. Nevertheless, the physics which drive the dispersion, namely the interplay between nuclear burning and hydrodynamical instabilities, is correctly taken into account in the model, as the parameter studies in Iapichino et al. (2006) show. Therefore one can reasonably expect that the numerical dispersion mimics on more extended length scales the effect of the cooling by heat diffusion at unresolved scales.

One can still argue about other oversimplifications in the bubble setup. For example, the choice of a spherical bubble

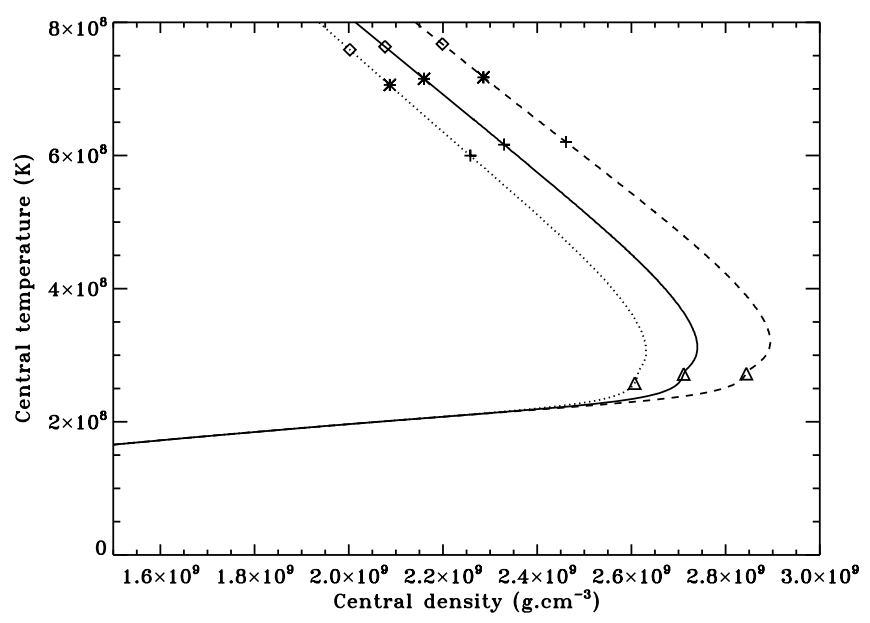

Fig. 4. Evolution for the central temperature and density of the star in Models 3 (solid), Model 3.1 (dashed) and Model 3.2 (dotted). Symbols denote the birth of the convective core (triangles) and ignition points according to various criteria: the burning time scale is $1 / 22$ nd of (crosses) $1 / 8$ th of (stars) or equal to (diamonds) the convective turnover timescale (see Lesaffre et al. 2006). Although the position of the crosses and stars corresponds to actual output models of the stellar evolution code, the diamonds result from an extrapolation involving models at a lower temperature.

shape can be debated. Numerical tests were performed with bubbles in stirred backgrounds, where the bubble is soon deformed and the growth of instabilities follows a different pattern with respect to the standard background model at rest (Iapichino et al. 2006). The results show that for well-posed choices of the stirring field the turbulence-induced disruption (and thus the bubble shape) has no leading effect in the bubble evolution.

Closely related to the bubble shape is the geometry choice: the 2D Cartesian geometry has been preferred to the more natural cylindrical, axisymmetric geometry for numerical reasons, after we verified that the results on bubble dispersion are very similar in both cases (Iapichino 2005).

Another numerical issue concerns the discretization of the bubble on the Cartesian grid. This procedure may introduce errors, potentially able to sow instabilities that can ultimately dominate the evolution. A resolution study has therefore been performed (Iapichino et al. 2006) to verify whether the chosen level of resolution allows for an adequate modeling of the hydrodynamical instabilities and the subsequent fragmentation. In Fig. 5 one can notice that different maximum levels of refinement resolve more and more Rayleigh-Taylor unstable modes. On the other hand, the resolution studies of the temperature evolution and the bubble dispersion (Figs. 3 and 4 of Iapichino et al. 2006) clearly have convergent results for the two more resolved runs, thus indicating that the bubble evolution is dominated by largescale instabilities rather than by discretization errors.

\section{Discussion and conclusions}

In this work we explored two previously unaccounted for parameters of the ignition theory of SNe Ia, and studied how they affect the features of the ignition process. The tools for this study are 2D numerical simulations of temperature perturbations in the WD core. In this framework, the bubble model developed by Iapichino et al. (2006) has several intrinsic and numerical limitations in its predictive power of the ignition properties of $\mathrm{SNe} \mathrm{Ia}$, 


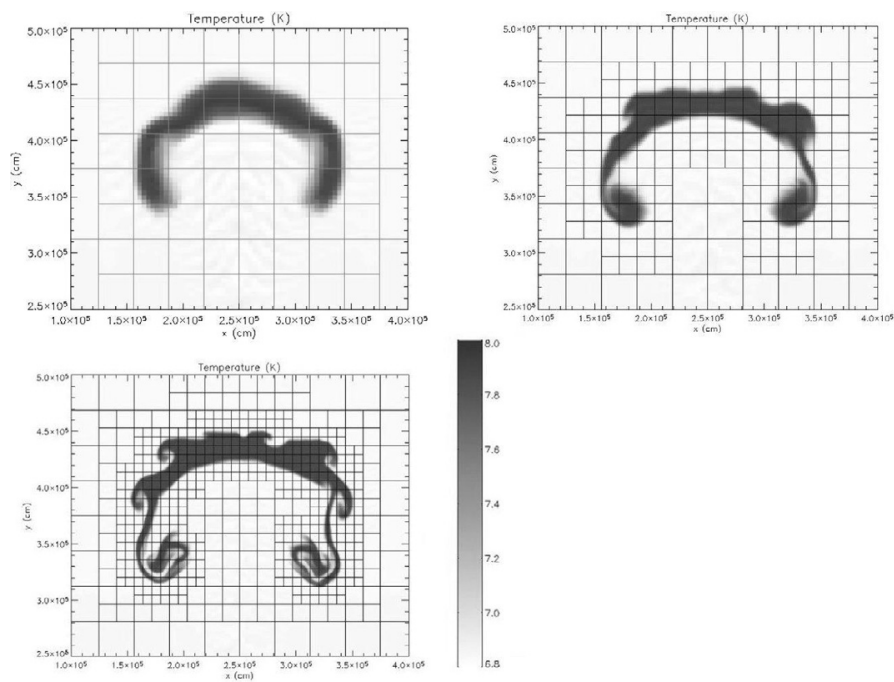

Fig. 5. Comparison of three temperature plots of buoyant bubbles, after a simulation time of $0.5 \mathrm{~s}$, in runs with different maximum levels of refinement. The plots enclose only a small part of the computational domain $(3 \times 2.5 \mathrm{~km})$, centered on the bubble. The choice of the initial parameters is identical in the three cases: temperature $7.7 \times 10^{8} \mathrm{~K}$, diameter $1 \mathrm{~km}$, distance from the center $100 \mathrm{~km}$, and the background WD model is the same as in Iapichino et al. (2006). Every mesh element in the plots is resolved in $8 \times 8$ grid cells. The color scale indicates the temperature in units of $10^{8} \mathrm{~K}$. The panels refer to runs with four (upper left), five (upper right; this is the reference resolution of the runs presented in this work) and six (lower left) AMR levels.

but it can be profitably used as a "probe" of the influence on the ignition process of other physical parameters ${ }^{2}$.

Four WD models, derived by the stellar evolution and accretion history of the progenitors (Lesaffre et al. 2006), were used as background for the bubble evolution. The thermonuclear runaway is not reached in the coolest and densest WD models 4 and 6, which result from the evolution of initially more massive progenitors. We infer that in these models the ignition region lies closer to the WD center where the gravitational acceleration is smaller and the bubble dispersion less effective.

The simplified bubble model that we used does not allow an accurate determination of the extent of the ignition zone. An estimate of this quantity is provided by the size of the burning zone radius $r_{\mathrm{b}}$, where one-half of the nuclear energy of the WD is produced and where the temperature perturbations are likely to be generated. The burning zone radius is in the range between 170 and $120 \mathrm{~km}$ and decreases in more massive progenitors, thus supporting the results of bubble simulations.

Clearly these estimates totally neglect any superimposed feature of the WD convection like a dipole. In large-scale simulations of the progenitor's hydrostatic evolution on the other hand the spatial resolution is insufficient to resolve the generation and ignition (or fragmentation) of the temperature perturbations. Therefore we suggest to implement the key properties of these objects using a stochastic approach (cf. Schmidt \& Niemeyer 2006).

The second question addressed by this work are the nuclear physics uncertainties in the ignition theory. Starting from the new measurement of Spillane et al. (2007), we study the impact

\footnotetext{
${ }^{2}$ For example, following the same approach, Iapichino et al. (2008) propose that the WD composition affects also the ignition features (as suggested by Dursi \& Timmes 2006) and not only the SNe Ia explosion (cf. Timmes et al. 2003; Röpke et al. 2006a; Townsley et al. 2009).
}

of a different (larger) reaction rate for the C-burning on the ignition features in SNe Ia. This analysis is not limited to the temperature perturbations, but is extended to the WD structure by computing new stellar evolution tracks with various rates of Cburning. According to these models, the ignition features only have a mild dependence on the rate at low temperature (where lower rates favor higher ignition densities) and almost no dependence on the rate at high temperature. A somewhat similar analysis has been performed by Cooper et al. (2009): they study the possible effect of a hypothetical resonance at $E=1.5 \mathrm{MeV}$ by constructing ignition curves for the progenitor. They also find that this feature would play no major role in the ignition process, though lower-lying resonances could. It is important to stress that the effect of different ignition conditions is manifold and not merely related to the location of the ignition zone; Cooper et al. (2009) for example conjecture an impact on the iron-peak explosive nucleosynthesis (Piro \& Bildsten 2008; Chamulak et al. 2008) and on the velocity of the flame front (Röpke et al. 2006a).

The whole field of the theoretical modeling of SNe Ia has reached in recent years a mature stage with growing interesting insights in the microphysics of these events (like for example the mechanism for DDT, Röpke \& Niemeyer 2007; Röpke 2007; Woosley 2007). Moreover, modern simulations provide more and more detailed comparisons with the observables (Hillebrandt et al. 2007; Mazzali et al. 2007; Kasen \& Woosley 2007). The use of SNe Ia for precision cosmology has finally found safer grounds thanks to a deeper theoretical understanding of the involved physical parameters (Röpke et al. 2007a; Kasen et al. 2009). At the same time, novel low-Mach number schemes permit to fill the gap between the evolution of the progenitor and the onset of the explosion (Zingale et al. 2008, 2009). In this context, the present study on the ignition parameters, although performed with very simplified tools, can drive further and more sophisticated analysis towards interesting issues, which call for a deeper investigation.

Acknowledgements. Thanks to the referee for the constructive comments, which improved the presentation of this work. The FLASH code is developed by the DOE-supported ASC / Alliance Center for Astrophysical Thermonuclear Flashes at the University of Chicago. L.I. thanks the organizers of the Catania Workshop on Nuclear and Neutrino Astrophysics (WNNA 2007) for the fruitful meeting, which inspired part of this work. P.L. thanks the French embassy in the United Kingdom for an overseas fellowship at Churchill College during which part of this work was conducted.

\section{References}

Caughlan, G. R., \& Fowler, W. A. 1988, Atomic Data and Nuclear Data Tables, 40,283

Chamulak, D. A., Brown, E. F., Timmes, F. X., \& Dupczak, K. 2008, ApJ, 677, 160

Cooper, R. L., Steiner, A. W., \& Brown, E. F. 2009, ApJ, 702, 660

Cussons, R., Langanke, K., \& Liolios, T. 2002, EPJ A, 15, 291

Dursi, L. J., \& Timmes, F. X. 2006, ApJ, 641, 1071

Fryxell, B., Olson, K., Ricker, P., et al. 2000, ApJS, 131, 273

García-Senz, D., \& Bravo, E. 2005, A\&A, 430, 585

García-Senz, D., \& Woosley, S. E. 1995, ApJ, 454, 895

Gasques, L. R., Brown, E. F., Chieffi, A., et al. 2007, Phys. Rev. C, 76, 035802 Hillebrandt, W., \& Niemeyer, J. C. 2000, ARA\&A, 38, 191

Hillebrandt, W., Sim, S. A., \& Röpke, F. K. 2007, A\&A, 465, L17

Höflich, P., \& Stein, J. 2002, ApJ, 568, 779

Iapichino, L. 2005, Ph.D. Thesis, Technische Universität München, Germany

Iapichino, L., Brüggen, M., Hillebrandt, W., \& Niemeyer, J. C. 2006, A\&A, 450, 655

Iapichino, L., Brüggen, M., Hillebrandt, W., \& Niemeyer, J. C. 2008, in Proceedings of the Catania workshop on nuclear and neutrino astrophysics, ed. E. Migneco, C. Spitaleri, \& R. A. Zappalá (INAF - Catania astrophysical observatory special publication), 101, [arXiv:0711.2027]

Itoh, N., Tomizawa, N., Wanajo, S., \& Nozawa, S. 2003, ApJ, 586, 1436 
L. Iapichino and P. Lesaffre: Uncertainties and robustness of the ignition in $\mathrm{SNe} \mathrm{Ia}$

Jiang, C. L., Rehm, K. E., Back, B. B., \& Janssens, R. V. F. 2007, Phys. Rev. C, 75,015803

Kasen, D., \& Woosley, S. E. 2007, ApJ, 656, 661

Kasen, D., Röpke, F. K., \& Woosley, S. E. 2009, Nature, 460, 869

Kuhlen, M., Woosley, S. E., \& Glatzmaier, G. A. 2006, ApJ, 640, 407

Lesaffre, P., Podsiadlowski, P., \& Tout, C. A. 2005a, MNRAS, 356, 131

Lesaffre, P., Podsiadlowski, P., \& Tout, C. A. 2005b, Nucl. Phys. A, 758, 463

Lesaffre, P., Han, Z., Tout, C. A., Podsiadlowski, P., \& Martin, R. G. 2006, MNRAS, 368, 187

Livne, E., Asida, S. M., \& Höflich, P. 2005, ApJ, 632, 443

Mazzali, P. A., Röpke, F. K., Benetti, S., \& Hillebrandt, W. 2007, Science, 315, 825

Nomoto, K. 1982, ApJ, 253, 798

Piro, A. L., \& Bildsten, L. 2008, ApJ, 673, 1009

Piro, A. L., \& Chang, P. 2008, ApJ, 678, 1158

Röpke, F. K. 2007, ApJ, 668, 1103

Röpke, F. K., \& Niemeyer, J. C. 2007, A\&A, 464, 683

Röpke, F. K., Gieseler, M., Reinecke, M., Travaglio, C., \& Hillebrandt, W. 2006a, A\&A, 453, 203

Röpke, F. K., Hillebrandt, W., Niemeyer, J. C., \& Woosley, S. E. 2006b, A\&A, 448, 1
Röpke, F. K., Hillebrandt, W., Schmidt, W., et al. 2007a, ApJ, 668, 1132

Röpke, F. K., Woosley, S. E., \& Hillebrandt, W. 2007b, ApJ, 660, 1344

Schmidt, W., \& Niemeyer, J. C. 2006, A\&A, 446, 627

Spillane, T., Raiola, F., Rolfs, C., et al. 2007, Phys. Rev. Lett., 98, 122501

Timmes, F. X., Hoffman, R. D., \& Woosley, S. E. 2000, ApJS, 129, 377

Timmes, F. X., Brown, E. F., \& Truran, J. W. 2003, ApJ, 590, L83

Townsley, D. M., Jackson, A. P., Calder, A. C., et al. 2009, ApJ, 701, 1582

Umeda, H., Nomoto, K., Yamaoka, H., \& Wanajo, S. 1999, ApJ, 513, 861

Woosley, S. E. 2007, ApJ, 668, 1109

Woosley, S. E., Kasen, D., Ma, H., et al. 2008, in Proceedings of the 10th Symposium on Nuclei in the Cosmos (NIC X). Mackinac Island, Michigan, USA. Available online at http://pos.sissa.it/cgi-bin/ reader/conf. cgi? confid $=53$

Woosley, S. E., Wunsch, S., \& Kuhlen, M. 2004, ApJ, 607, 921

Wunsch, S., \& Woosley, S. E. 2004, ApJ, 616, 1102

Zingale, M., Almgren, A. S., Bell, J. B., Malone, C. M., \& Nonaka, A. 2008, J. Phys. Conf. Ser., 125, 012013

Zingale, M., Almgren, A. S., Bell, J. B., Nonaka, A., \& Woosley, S. E. 2009, ApJ, 704, 196

Zingale, M., \& Dursi, L. J. 2007, ApJ, 656, 333 